\title{
Analysis of different clinical and biological Factors affecting Pregnancy Rate in frozen-thawed embryo transfer
}

\author{
Nahed Hussain Mohammed*- \\ Ahmed Mohammad Rammah** \\ Hanaa Fathi Abu Ria* \\ - Abdel Aal El Ghandour** - \\ Ahmed Gamal Serour** . \\ Eman Anwar Hassan** \\ *Faculty of Medicine For Girls, \\ Al-Azhar University. \\ **International Islamic Centre \\ for Population Studies and \\ Researches(IICPR) Al-Azhar University
}

Corresponding author:

Dr.Eman Anwar Hassan /

International Islamic Centre for

Population Studies and

Researches (IICPR) Al-Azhar

University, Cairo, Egypt.Tel: (+2)

01221354112.

emanembryology@gmail.com

\begin{abstract}
$\underline{\text { Abstract }}$
Frozen embryo transfer (FET) is an important part of present-day IVF/ ICSI treatment. There is limited understanding of the factors affecting success rates after FET. The aim of this work to study the Clinical and laboratory factors which affect clinical pregnancy rate after frozen-thawed embryo transfer.
\end{abstract}

Study design: This is three-center prospective study. Analysis was carried out on 150 consecutive FET cycles during the period from 2011 - 2015. The primary outcome was clinical pregnancy per cycle. We assessed the independent effect on clinical pregnancy of the following variables: female age, female age at embryo freezing, BMI, primary versus secondary infertility, cause \& duration of infertility, LH/FSH ratio, protocol of fresh cycle, serum estradiol level before Human Chronic Gonadotropin (HCG) at fresh cycle, sperm source (testicular or ejaculated), day of freezing, pregnancy in the fresh cycle, storage duration of embryos, cause of, thawing method as well as number of thawed transferred embryo (s).

Main results: Pregnancy rate in FET was 31\%. Pregnancy rate was 21.6\% in patient younger than 30 years old at fresh cycle with RR 2.31 with CI (1.72-7.37). Patients of age at FET more than 35 years to have positive outcome $9.4 \%$ of FET with $95 \% \mathrm{CI}=(1.72-7.37)$. There was absolute risk increase of 2.91 between the two BMI groups $(>=30 \&<30)$ with $95 \%$ CI: (1.13-7.50) \%. patients having explained cause of infertility would be 0.26 times as likely as patients having unexplained infertility to have positive outcome of FET with $95 \% \mathrm{CI}=(0.12-0.55)$. Patients having abnormal semen analysis would be 1.44 times as likely as patients having normal analysis to have positive outcome of FET with $95 \% \mathrm{CI}=(0.20-$ 0.99). Patients having Negative Pregnancy outcome at fresh cycle would be 0.9 times as likely as patients having Positive Pregnancy outcome at fresh cycle but this is not significant $95 \% \mathrm{CI}=(0.38-2.66)$. Patients with excess number of embryos as a cause of freezing would be 0.38 times as likely as patients of unusual Cause of freezing to have positive outcome of FET $16.2 \%$ with $95 \% \mathrm{CI}=(0.14-0.99)$. The chance of pregnancy increases significantly if $\geq 3$ thawed embryos was transferred (odds ratio (OR) 0.34 95\% confidence interval (CI) (0.12-0.97).

Conclusion: Increased number of transferred thawed embryos is associated with increased clinical pregnancy; Age, BMI, cause of infertility, semen parameters, pregnancy at fresh cycle, endometrial thickness on the thawed embryo transfer day and cause of freezing. Number of transferred thawed embryos affected independently the clinical pregnancy rate in FET.

\section{Introduction}

The first IVF pregnancy was achieved in 1978. Since then, the number of IVF centers and IVF procedures performed has increased dramatically (1). In order to reduce the chance of multiple pregnancies during in-vitro fertilization (IVF)-ET treatment, there is an increasing trend towards 
transferring a lower number of embryos. Surplus good-quality embryos are then transferred in frozenthawed embryo transfer (FET) cycles. The first pregnancy after FET was reported in 1983. Elective cryopreservation of all fresh embryos is also required in special circumstances where fresh embryo transfer is undesirable. Examples of the latter include: patients in whom the risk of ovarian hyper stimulation syndrome (OHSS) is high, or those involving oocyte or embryo donations where a quarantine period is advisable (2).Several investigators have detected lower implantation and pregnancy rates in frozen thawed embryo replacement cycles compared with fresh IVF cycles, and two important etiologies of this lowered rate are adverse effect of the freeze thawing process $\&$ ice crystal damage and transferring higher-quality embryos in the fresh cycle and freezing the lowergrade ones. The question that comes to mind is whether there is any way to improve the pregnancy outcome of a cryopreserved embryo transfer cycle. A few studies have assessed the clinical and embryological factors affecting pregnancy outcome, and there is controversy about the parameters among the performed studies (3). In this study, data from frozen embryo transfer (FET) cycles was collected for a better understanding about some of the clinical and embryological factors that may influence pregnancy rate.

\section{$\underline{\text { Subjects and methods }}$}

Our study was conducted at the International Islamic Centre for Population Studies and Researches (IICPR) IVF unit and two private IVF centers in Cairo. It was performed during March 2011 to June 2015.The study was randomized control study collecting data from prospective cases who underwent frozen embryo transfer (FET) after ICSI procedure. We studied 150 couples complaining of infertility who have been undergone FET after fresh cycle. Female age more than 40 years old and poor responders patients were excluded from our study. The clinical factors data were collected consisting of maternal age at both oocyte retrieval and at time of FET, BMI, duration , type \& cause of infertility, sperm parameters, FSH / LH ratio, protocol of controlled ovarian cycle ,E2 at day of HCG, source of sperm, pregnancy outcome at fresh cycle, reasons for embryo cryopreservation, endometrial thickness on the day of embryo transfer, duration embryo cryopreservation and number of thawed- transferred embryos. Controlled ovarian stimulation $(\mathrm{COH})$ was achieved mainly using the gonadotropin-releasing hormone agonist $(\mathrm{GnRH})$ for pituitary suppression in the fresh cycle.
$\mathrm{COH}$ was done by $\mathrm{HMG}$ either through long protocol (in 96 cycles) or short protocol (in 54 cycles). The subjects undergo pituitary desensitization by a long acting GnRH analogue administered in the luteal phase of the previous cycle. While patients who submitted to short protocol were given GnRH agonist starting early in the follicular phase of ovulation induction cycle (cycle day 1) then exogenous gonadotropins starting on cycle day 2 an initial daily dose of $150 \mathrm{IU}$ HMG has been started. This dose is adjusted according to the patient's response after 6 days of stimulation as assessed by TVS and E2 level. Folliculometry and endometrial thickness evaluation were done using TVS. When follicles reach $18 \mathrm{~mm}$ or more, HCG (5000 or $10000 \mathrm{IU}$ ) was given. TVS directed oocyte recovery was performed 34-36 hours after HCG administration, in the operating theatre under full aseptic technique under light general anesthesia.

All semen parameters were recorded and evaluated in accordance to the WHO standards 2010 of semen evaluation. Sperms obtained from husband either through fresh ejaculation in $128(85.4 \%)$, testicular sperm extraction (TESE) in $15(10 \%)$ or percutaneous sperm aspiration (PESA) in 7 (4.6\%).The ICSI procedure involved the injection of a single motile spermatozoon into the cytoplasm of oocyte. The cleavage characteristics of the fertilized oocytes were evaluated: the numbers and sizes of blastomeres and the presence of a nucleate cytoplasmic fragment are recorded. The cleaving embryos are scored according to cleavage stage, blastomeric size and shape, and fragmentation. The assessment criteria of blastocyst quality were based on blastocoel cavity, inner cell mass, trophectodermal cells, and volume of zona-pellucida. Embryos with grade A were transferred on Day 2, 3,4,5 or 6 (where day of fertilization were considered day 1).Surplus embryos (with grade A or B) in fresh cycles were cryopreserved for subsequent FET cycles.

Freezing and thawing were performed with 1, 2-propanediol and sucrose as cry protectants. Straws were used for freezing and storage. Embryos were cryopreserved by vetrification in 139 cases $(92.6 \%)$ and by slow method in 11 cases $(10.1 \%)$. The embryos are thawed either the day before (if blastocyst) or on the day of the scheduled embryo transfer (cleavage stage). Embryo thawing takes approximately 2 hours. Sometimes individual cells within the embryo were damaged by the freezing process. Embryos with some freezing damage can still go on to produce a healthy pregnancy however the more the embryo is damaged the less likely it will be for the embryo to develop. Sometimes all of the cells within the embryo were damaged and was not been transferred. Embryo 
quality was typically evaluated at two stages: just before freezing and about 4 hours before transfer. An additional evaluation was also performed immediately after thawing. Embryos were transferred if there are $\geq 50 \%$ intact blastomeres and no multinucleated blastomeres were observed after thawing. The embryos that would be transferred were loaded into the ET catheter. Labotect catheter: $150 \mathrm{~mm}$ long, a traumatic catheter and having a pre-curved guiding cannula with spherical finish. The hormonal replacement therapy (HRT) protocol was used for FET. Oral estradiol (2 $\mathrm{mg} / \mathrm{d}$ ) was taken daily from cycle day 5 . Transvaginal ultrasound scan was performed at eleventh day of the cycle to assess endometrial-thickness. Progesterone (intramuscularly or as vaginal suppository ) was given when the endometrium reached a thickness $>$ $8 \mathrm{~mm}$. FET was done 2,3,4,5 or 6 days after starting progesterone according to day of freezing. Both estrogen \& progesterone were continued for 2 weeks, if pregnancy test was positive they were continued until 8 weeks gestation. Patients (who were not fasting and with full urinary bladder) were put in lithotomy position frozen embryo transfer, and was considered as positive biochemical pregnancy. Clinical pregnancy and abdominal ultrasound guided embryo transfer was done using Labotect ET catheter. Confirmation of a successful implantation was performed by detecting serum human chorionic gonadotropin ( $\beta$-HCG) concentration $(>25 \mathrm{IU} / \mathrm{ml}) 2$ weeks post- was defined by a presence of a gestational sac, fetal pole and fetal heart activity by ultrasound at 7 weeks of pregnancy. Overall success rate were calculated and factors affecting incidence of clinical pregnancy after FET were analyzed.

\section{Statistical analysis}

Different specific software packages were used through- out the analysis. They are: EGRET, EPIINFO 7.1.5, EPI-INFO 3.5.1, DATA 6.1, SPSS ver.19 and Excel 2010. Of normally distributed continuous variables were assessed by Student's t test. Significant differences were evaluated by the Chi-square test to compare the non- continuous variables. The data were expressed as mean $\pm \mathrm{SD}$. P-value $<0.05$ was considered statistically significant.

\section{Results}

Table 1: shows the background characteristics of the studied cycles

\begin{tabular}{|l|c|c|}
\hline \multicolumn{1}{|c|}{ Risk Factor } & Mean & S.D \\
\hline Age & 27.37 & 4.78 \\
\hline BMI & 29.50 & 5.78 \\
\hline Infertility duration & 5.07 & 3.20 \\
\hline LH /FSH ratio & 0.80 & 0.44 \\
\hline Prolactin & 17.32 & 8.71 \\
\hline Basal E2 & 44.41 & 37.59 \\
\hline E2on day of HCG & 2610.06 & 1036.37 \\
\hline No. of retrieved Oocytes & 13.21 & 3.37 \\
\hline Total no. of embryos (grade A) & 2.75 & 0.13 \\
\hline No. of ET. At fresh cycle & 2.77 & 0.56 \\
\hline Total no of cryopreserved embryos (grade A) & 2.56 & 0.26 \\
\hline Time to FET & 3.45 & 1.36 \\
\hline Age at FET & 30.56 & 2.36 \\
\hline NO. OF thawed ET & 3.15 & 1.80 \\
\hline Day of freezing & 2.10 & 2.10 \\
\hline & $($ Median=3.00) & $($ Mode=3.00) \\
\hline
\end{tabular}

On average, FET cycles were performed 1.5 year after cryopreservation. The overall PR in all FET cycles was $18 \%$ (27 patients). 
Table 2: Shows the female clinical factors influencing clinical pregnancy rates.

\begin{tabular}{|c|c|c|c|c|c|}
\hline \multicolumn{2}{|c|}{ Clinical factors } & \multirow{3}{*}{\begin{tabular}{|c|} 
No of patients \\
94 \\
56 \\
\end{tabular}} & \multirow{3}{*}{$\begin{array}{c}\begin{array}{c}\text { Clinical } \\
\text { pregnancy rate } \\
(\%)\end{array} \\
21.6 \% \\
9.4 \% \\
\end{array}$} & \multirow{3}{*}{$\begin{array}{c}\begin{array}{c}\text { Relative Risk } \\
\text { (RR) }\end{array} \\
2.31\end{array}$} & \multirow{3}{*}{$\begin{array}{c}\begin{array}{c}\mathbf{9 5 \%} \% \\
\text { Confidence }\end{array} \\
\text { interval (CI) }\end{array}$} \\
\hline \multirow{2}{*}{ Age (years) } & $<30$ & & & & \\
\hline & $\geq 30$ & & & & \\
\hline \multirow{2}{*}{$\begin{array}{c}\text { BMI } \\
\mathrm{Kg} / \mathbf{m} 2\end{array}$} & $\geq 30$ & 72 & $21.7 \%$ & \multirow{2}{*}{2.91} & \multirow{2}{*}{$1.13-7.50$} \\
\hline & $<30$ & 78 & $9.3 \%$ & & \\
\hline \multirow{2}{*}{ Type of Infertility } & primary & 89 & $19.1 \%$ & \multirow{2}{*}{1.62} & \multirow{2}{*}{$-0.41-6.39$} \\
\hline & secondary & 61 & $11.9 \%$ & & \\
\hline \multirow{2}{*}{$\begin{array}{l}\text { Cause of } \\
\text { infertility }\end{array}$} & Explained & 97 & $14.4 \%$ & \multirow{2}{*}{0.26} & \multirow{2}{*}{$0.12-0.55$} \\
\hline & Unexplained & 53 & $17.6 \%$ & & \\
\hline \multirow{2}{*}{$\begin{array}{c}\text { Duration of } \\
\text { infertility(years) }\end{array}$} & $>5$ ys & 81 & $21.6 \%$ & \multirow{2}{*}{1.36} & \multirow{2}{*}{$0.60-3.07$} \\
\hline & $<=5$ & 69 & $9.4 \%$ & & \\
\hline \multirow{2}{*}{ LH/FSH ratio } & $\geq 1$ & 73 & $24.1 \%$ & \multirow{2}{*}{1.55} & \multirow{2}{*}{$0.68-3.55$} \\
\hline & $<1$ & 77 & $6.9 \%$ & & \\
\hline \multirow{2}{*}{ Protocol of $\mathrm{COH}$} & Long & 96 & $16.7 \%$ & \multirow{2}{*}{0.56} & \multirow{2}{*}{$0.20-1.58$} \\
\hline & Short & 54 & $14.3 \%$ & & \\
\hline \multirow{2}{*}{$\begin{array}{c}\text { E2 at day of } \\
\text { HCG2 } \\
\text { Pg/ml }\end{array}$} & $<=3500$ & 88 & $18.2 \%$ & \multirow{2}{*}{1.09} & \multirow{2}{*}{$0.35-3.36$} \\
\hline & $>3500$ & 52 & $12.8 \%$ & & \\
\hline
\end{tabular}

Table 3: shows the male factors influencing clinical pregnancy rates.

\begin{tabular}{|c|c|c|c|c|c|}
\hline \multicolumn{2}{|c|}{ Sperm sources } & \multirow{2}{*}{$\begin{array}{c}\text { No of patients } \\
71 \\
\end{array}$} & \multirow{2}{*}{$\begin{array}{c}\text { Clinical } \\
\begin{array}{c}\text { pregnancy rate } \\
(\%)\end{array} \\
9.0 \% \\
\end{array}$} & \multirow{2}{*}{$\begin{array}{c}\begin{array}{c}\text { Relative Risk } \\
\text { (RR) }\end{array} \\
1.44\end{array}$} & \multirow{2}{*}{$\begin{array}{c}\begin{array}{c}95 \% \\
\text { Confidence } \\
\text { interval }(\mathbf{C I})\end{array} \\
0.20-0.99 \\
\end{array}$} \\
\hline \multirow{2}{*}{ Semen } & Abnormal & & & & \\
\hline & Normal & 57 & $13.8 \%$ & 0.56 & $0.21-0.93$ \\
\hline Testicular & PESA\&TESE & 22 & $8.2 \%$ & 1.02 & $0.38-2.66$ \\
\hline
\end{tabular}


Table 4: shows the clinical factors influencing clinical pregnancy rates.

\begin{tabular}{|c|c|c|c|c|c|}
\hline \multicolumn{2}{|c|}{ Clinical factors } & No of patients & $\begin{array}{c}\text { Clinical } \\
\text { pregnancy rate }\end{array}$ & $\begin{array}{l}\text { Relative Risk } \\
\text { (RR) }\end{array}$ & $\begin{array}{c}95 \% \\
\text { Confidence }\end{array}$ \\
\hline \multirow{2}{*}{ Day of freezing } & Cleavage stage & 110 & $17.0 \%$ & \multirow{2}{*}{0.51} & \multirow{2}{*}{$0.15-1.71$} \\
\hline & Blastocyst & 40 & $14.0 \%$ & & \\
\hline \multirow{2}{*}{$\begin{array}{c}\text { Pregnancy } \\
\text { outcome from } \\
\text { fresh cycle }\end{array}$} & Negative & 85 & $16.0 \%$ & \multirow{2}{*}{0.90} & \multirow{2}{*}{$0.38-2.15$} \\
\hline & Positive & 65 & $18 \%$ & & \\
\hline \multirow{2}{*}{$\begin{array}{c}\text { Duration of } \\
\text { cryopreservation }\end{array}$} & $\leq 1 \mathrm{y}$ & 67 & $20.9 \%$ & \multirow{2}{*}{1.63} & \multirow{2}{*}{$0.64-4.18$} \\
\hline & $>1 y$ & 83 & $10.1 \%$ & & \\
\hline \multirow{2}{*}{ Cause of freezing } & Excess embryos & 99 & $16.2 \%$ & \multirow{2}{*}{0.38} & \multirow{2}{*}{ 0.14-0.99 } \\
\hline & Other cause & 51 & $15.8 \%$ & & \\
\hline \multirow{2}{*}{ Thawing method } & Slow & 11 & $10.1 \%$ & \multirow{2}{*}{1.02} & \multirow{2}{*}{$0.27-3.82$} \\
\hline & Vetrification & 139 & $20.9 \%$ & & \\
\hline \multirow{2}{*}{ Age at FET } & $>35$ & 56 & $9.4 \%$ & \multirow{2}{*}{0.35} & \multirow{2}{*}{$1.08-3.59$} \\
\hline & $<=35$ & 94 & $21.6 \%$ & & \\
\hline \multirow{2}{*}{$\begin{array}{c}\text { No. of Thawed } \\
\text { Transferred } \\
\text { Embryos }\end{array}$} & $<3$ & 58 & $13.5 \%$ & \multirow{2}{*}{0.48} & \multirow{2}{*}{$0.22-0.89$} \\
\hline & $\geq 3$ & 92 & $17.5 \%$ & & \\
\hline
\end{tabular}

Table 5: Multivariate logistic regression analysis for pregnancy outcome

\begin{tabular}{|c|c|c|}
\hline NO. OF thawed ET (YES/NO) & p-value & OR(95\% $\mathbf{C I})$ \\
\hline$\geq \mathbf{3}$ & 0.0445 & Reference Category \\
$0.34(0.12-0.97)$
\end{tabular}

As noted, the pregnancy rate after FET in women aged $<35$ years was $21.6 \%$; In contrast, the pregnancy rate in patients aged $>35$ years was $9.4 \%$. Statistical analysis showed that young $(<35)$ and old $(35-40)$ mothers have significant differences in pregnancy rate following FET (95\% CI: 1.72-7.37). In our study, pregnancy rate was $31 \%$. cryopreservation was done for OHSS\& endometrial polyp in 51 cases which their pregnancy rate was $15.8 \%$. Pregnancy rate in short protocol subjects (54 cases) were 14.3\% that showed statistically insignificant difference to long protocol patients (96 cases) who had $16.7 \%$ positive clinical pregnancy. Concerning to the BMI, our results indicated a difference between patients $=>30 \mathrm{~kg} /$ m2 BMI (72 cases) with pregnancy rate $21.7 \%$, when compared to patients $<30$ BMI (78 cases) with $9.3 \% \%$ of pregnancy rate. Pregnancy rate in women, who had unexplained cause of infertility, showed a significant difference in comparison to the subjects with explained cause $(95 \% \mathrm{CI} 0.12-0.55)$. Couples with normal semen parameters had pregnancy rate $13.3 \%$ compared to couples with abnormal semen who had pregnancy rate $9.0 \%$. There was no pregnancy in patients with endometrial thickness $<8 \mathrm{~mm}$ on FET day. By logistic regression analysis, the most detector factor for pregnancy outcome was number of thawed embryo transfer (table 5). Our data showed that the some clinical factor such as primary or secondary and duration of infertility, LH/FSH ratio, type of protocol, level of E2 at day of HCG, source of sperm, day of freezing, pregnancy outcome at fresh cycle, duration of cryopreservation and thawing method had no influence on pregnancy outcomes. The detailed data are presented in tables. 


\section{$\underline{\text { Discussion }}$}

In this study we attempted to evaluate prognostic factors associated with pregnancy outcome in frozen embryo transfer (FET). We carried out an analysis of 150 cases of FET and identified 18 significant clinical variables. These factors are: age of female at both fresh \&FET cycles, BMI, type, cause and duration of infertility, semen parameters, LH $\backslash F S H$ ratio , serum prolactin, basal E2, protocol of fresh cycle ,E2 at day of HCG, sperm source, day of freezing, total number of cryopreserved embryos, pregnancy outcome at fresh cycle, time interval between fresh and FET cycles, cause of freezing, thawing method, endometrium thickness at FET and number of thawed transferred embryos .The age of the women has generally been considered to be an important determinant of treatment success following both fresh and frozen embryo transfers. Another study (2) showed that women aged 35 years or below had higher pregnancy rates than older women. Many studies showed some common characteristics in women above 30 , especially above 35 , include diminishing ovarian reserve, poor quality of remaining oocytes, decreasing endometrial receptivity and abnormal genetic factors (4). By analysis of our collected data It was obvious that patients aged $<30$ years old would be 2.3 times as likely as patients aged $>=30$ years old to have positive outcome of FET. Where pregnancy rate was $21.6 \%$ in patients aged $<30$ while it was only $9.4 \%$ patients aged $>=30$ years. Also age at FET is an important factor where patients of age at $\mathrm{FET}<=35$ would be 1.88 times as likely as patients of age at FET $>35$ to have positive outcome of FET. Many studies support our results and found that with advanced age, the pregnancy rate reduced after frozen embryo transfer, and the major reduction occurred in women above 40 years $(4 \& 5)$. But these contradict to a study (3), in which they have found no impact of women age on FET success rates; they concluded that the quality of embryos was crucial factor determining the success of FET. Also another studies $(6,7)$ did not find significant differences in pregnancy outcome in two maternal age groups (40 years and $>40$ years) and suggested that if enough good-quality eggs and embryos were transferred, the age does not have an effect on pregnancy outcome. Obesity contributes to bad outcomes following treatment using assisted reproductive technology, including lower pregnancy and a higher miscarriage rate and live birth rates. This could be mediated through a detrimental effect on oocyte and embryo quality (8). Another study (9) analyzed frozen-thawed embryo transfers cycles and conclude that obesity negatively impact pregnancy rates. On the other hand, $(3,10)$ in another studies found that no significant difference was found in BMI between pregnant and none pregnant groups. (3).On the other hand Bellver's study (11) compared embryo quality and reproductive outcome among BMI groups and conclude that female obesity impairs ICSI outcome, but embryo quality is not affected, pointing to an alteration in uterine environment. This explains our results that BMI $>=30$ usually have $\mathrm{PCO}$ patient have more oocyte retrieved and good quality embryos. So, by improving uterine environment using HRT regimen, the outcome would be improved. Where patients having BMI $>=30$ would be 2.91 times as likely as patients having BMI $<30$ to have positive outcome of FET with $95 \% \mathrm{CI}=(1.13-7.50)$. In addition this BMI was measured at fresh cycle and it might be corrected later on in FET cycle.

In our study we found that types of infertility either primary or secondary and durations of infertility had no significance effect on pregnancy outcome after FET. This is supported by study of Eftekhar (12) who concluded that the type and duration of infertility seems to have no effects on the pregnancy outcome following FET. This indicates that wherever good embryos are present, the chance of pregnancy is increased. In our study, patients who had unexplained cause of infertility had more positive outcome than patient who had underlying factor. Our results may be explained on the basis of those patients with explained infertility usually referred to ICSI at earlier age. Another study $(7,13)$ did not find an association between cause of infertility and clinical outcomes in FET.

In our study, Patients having abnormal semen was 1.44 times as likely as patients having normal semen parameters to have positive outcome of FET. The question of whether male factor infertility would affect the outcome of ICSI and the health of the offspring has gained great attention since the introduction of ICSI for the treatment of patients with extremely poor-quality sperm (14). Although ICSI can help sperm penetrate the zona pellucida and reach the cytoplasm of the oocytes, the completion of oocyte activation and the formation of the male and female pronuclei are determined by the intrinsic nature of the sperm and oocytes $(15,16$ and 17) Many studies investigate the effect of different sperm qualities on the clinical outcome and perinatal parameters of offspring and concluded that there is no significant effect when different qualities and sources of sperm are used for ICSI $(18,19$, and 20). However till now no study investigates the effect of sperm parameters on outcome of FET.

In our study there was no statistically significant difference in outcome between patients with different 
sperm origin. This is supported by (6) study that concluded that the sperm origin did not affect the outcome of frozen-thawed embryo transfer cycles. We studied the effect of FSH $\backslash \mathrm{LH}$ ratio on pregnancy rate of FET and concluded that although patients of LH / FSH ratio $\geq 1$ would be of 1.55 as likely as patients of $\mathrm{LH} / \mathrm{FSH}$ ratio $<1$ to have positive outcome of FET, it is not statistically significant. LH is required for normal folliculogenesis, while low LH concentrations on day 3 could lead to a poor ovarian response (21). At the beginning of stimulation, high LH concentration can lead to increased endometrial maturation at oocyte pick-up (22).However, basal LH and E2 levels are not considered as proper factors in order to distinguish the infertile patients responding differently to ovarian stimulation (23). Other studies also revealed the FSH level caused a remarkable effect on pregnancy rate in FET (24, 25, 26 and 3). In another investigation, Kassab's study (27) reported inverse correlation between basal serum FSH levels before fresh IVF/ICSI cycle with pregnancy outcome in FET cycles.

As regard the type of $\mathrm{COH}$ protocol at fresh cycle, we didn't find any difference in outcome of FET between short and long protocol. This is similar to study (12) that concluded that ovulation-stimulating protocol has no effects on the pregnancy outcome following FET. Generally, after stimulation with the GnRH agonist long protocol, a large amount of oocytes are obtained in women with normal ovaries, and large numbers of embryos are provided for fresh embryo transfer or freezing. Consequently, significantly lower cancellation and higher pregnancy rates are observed in the long protocol in comparison with those of other stimulation protocols (28). Another study (29) and (7) reported higher pregnancy and implantation rates in women stimulated with the long protocol than those in women with short protocol in FET cycles. In our study, there was no statistically significant difference in outcome of FET between patients with different E2 levels at day of HCG in fresh cycle. This is supported by another study $(30,31)$ concluded that cryo-survival of frozen embryos are equal in both patients who have a normal stimulation profile and patients with OHSS.

In our study, we didn't find any difference in outcome of cleavage stage and blastocyst in FET. This is supported by study in which, they concluded that day 3 and 5 transfer had similar pregnancy and implantation rates (32). This explained by the fact that good quality embryo present is the most important factor of good outcome. This contradicts with other studies that showed improved success rates with transfer of blastocysts compared with cleavage-stage $(33,34$ and 35$)$. In our study patients with negative pregnancy was 0.9 times as likely as patients with positive pregnancy from fresh cycle but this result was not statistically significant. Pregnancy in the fresh ICSI cycle has been shown to be associated with an improved PR in subsequent FET in many studies (36 and 7). Conversely, a study has reported impaired implantation rate in FET for patients who became pregnant in their fresh treatment cycle (37).

In this study, although we found that embryos with duration of cryopreservation equal or less than one year gave better outcomes, this was not statistically significant. Also another study showed no significant difference in pregnancy outcome according to the duration of cryopreservation. In our study patients who had FET due to specific cause like polyp or OHSS had more positive outcome (7). This differs from study of Eftekhar in which they found that the cause of embryo cryopreservation has no effects on the pregnancy outcome following FET (12). Currently, vetrification does not appear to be associated with an increased probability of pregnancy. However, a significant advantage of vetrification over slow freezing in terms of post-thawing survival rates is present (38). This support our study where there is no significant difference between pregnancy rate in both verification and slow method. But there was large decrease in post-thawing survival rates with slow method due to degenerated embryos.

In our study we investigated the association between endometrial thickness and treatment outcome in HRT cycles. Endometrial thickness was regarded as prognostic parameters for successful pregnancy in FET. There was no positive pregnancy in patients with endometrial thickness $(<8 \mathrm{~mm})$. This is supported by study $(36,7)$ while contradicts (26) and (12) in their studies in which they concluded that endometrial thickness at embryo transfer day had not influence on the pregnancy rate after FET. Also other results concur with the consensus that there is no endometrial thickness value that precludes pregnancy (39).

In the present study patients having number of thawed transferred embryos $>=3$ have more positive outcome of FET. A higher number of FETs was associated with a significant increase in rates of clinical pregnancy, ongoing pregnancy, and multiple pregnancy. However, the transfer of more than one embryo should always be weighed against the risk of multiple pregnancies (40). According to the results of other investigators, there is a controversy among studies in the relationship between the number of embryos transferred and pregnancy outcome. Some researchers have reported a higher pregnancy rate with greater number of embryos transferred (41) and other studies did not support this relationship (3). 


\section{Conclusion}

In our study, we found that there were positive effect in FET of younger age, BMI $>30$, , unexplained cause of infertility, normal semen, endometrial thickness $>=$ $8 \mathrm{~mm}$ on FET day, cause of freezing other than excess embryos, increased number of thawed transferred embryos. By logistic regression analysis, the most detector factor for pregnancy outcome was number of thawed embryo transfer. On the other hand, there were no significant effect of type and duration of infertility, LH/FSH ratio, type of stimulation, level of E2 at day of HCG, origin of sperm, stage of freezing (cleavage or blastocyst), pregnancy outcome at fresh cycle, duration of cryopreservation and thawing method.

\section{References:}

1. Petrozza JC. Assisted Reproduction Technology htt: // emedicine. Med scape.com/ Downloaded Sep; 2013.

2. William SB, Raymond $\mathbf{H}$ W, Ernest $\mathrm{H} Y$ and Estella Y L. Frozen-thawed embryo transfer cycles. Hong Kong Med.J. 2009; 15:420-6.

3. Ashrafi M, Jahangiri N, Hassani F, Akhoond M R and Madani T. The factors affecting the outcome of frozen-thawed embryo transfer cycle. Taiwan J of Obs and Gynecology 2011; 50:159-164.

4. Lu L I , Xiao-xi SUN, Jun-ling CHEN, Xiao-Hong GAO, Yong-wei WANG, Jie-wei TAO, et al . Analysis of Factors Influencing Pregnancy Rate in Frozenthawed Embryo Transfer. J Reprod and Contra 2004; 15 (4):239-244.

5. Karlstrom P, Bergh T, Forsberg A, Sandkvist U. and Wikland M. Prognostic factors for the success rate of embryo freezing. Hum Reprod 2007; 12: 1263-6.

6. Kim J W , Byun H K, Youm H W , Park Y S, Song I $\mathbf{O}$, Song $\mathbf{J} \mathbf{H}$, et al. Analysis of Factors Affecting Survival and Pregnancy Rate in Frozen-thawed Embryo Transfers. Fertil Steril 2000; 27(1):59-66.

7. Mahnaz A, Shahideh J, Sadat M, Mohammad R, Firouzeh G, and Zahra Zolfaghari . ICSI Outcome in Infertile Couples with Different Causes of Infertility: A Cross-Sectional Study. Fertil Steril 2013; 7(2): 88-95.

8. Guy's and St Thomas. The impact of raised BMI on the outcome of assisted reproduction: Current concepts. J Obst and Gynecol 2011; 31(7):561-5.

9. Dessolle L, Emile D , Dominique C, Roman R , Charles C , Jacqueline M, et al. Determinants of pregnancy rate in the donor oocyte model: a multivariate analysis of 450 frozen-thawed embryo transfers. Hum Reprod 2009; 24(12):3082-3089.
10. Usoniene A, Eerkiene $\mathbf{P}$, and Usonyte A. Prognostic factors for clinical pregnancy using in vitro fertilization/ intracytoplasmic sperm injection and embryo transfer procedures. Acta Medica Lituanica 2004; 11(4):31-46.

11. Bellver J , Yanira M D , Marcos M D , Melo M ,Eduardo G, Antonio P,et al . Female obesity impairs in vitro fertilization outcome without affecting embryo quality. Fertil Steril 2010; 93(2):447-454.

12. Eftekhar M, Rahmani E, and Soheila Pourmasumi Evaluation of clinical factors influencing pregnancy rate in frozen embryo transfer. Iran J Reprod Med 2014; 12(7): 513-518.

13. Ye Xu B M, Jianhua Li, Guoping Zhou, Junhong Guo. Clinical outcomes for various causes of infertility with natural-cycle in vitro fertilization combined with in vitro maturation of immature oocytes. Gynecol Endocrinol 2010; 94(2):777-780.

14. Bonduelle M , Camus M, de Vos A, Staessen C, Tournaye H, van Assche E, et al . Seven years of intracytoplasmic sperm injection and follow- up of 1987 subsequent children, Hum Reprod 1999; 14(s1):243-264.

15. Perreault S D. Chromatin remodeling in mammalian zygotes. Mutat Res 1992; 296(1-2):43-55.

16. Schatten G. The centrosome and its mode of inheritance: the reduction of the centrosome during gametogenesis and its restoration during fertilization. Dev Biol 1994; 165(2):299-335.

17. Yanagimachi R. Mammalian Fertilization In: Knobil E, Neil JD (editors), Physiology of Reproduction, New York: Raven Press 1994; 189-317.

18. Yue-hong Lu, Hui-juan Gao, Bai-jia Li, Ying-ming Zheng, Ying- hui Ye, Yu-li Qian, et al. Different sperm sources and parameters can influence intracytoplasmic sperm injection outcomes before embryo implantation . J Zhejiang Univ Sci B 2012; 13(1): 1-1.

19. Ludwig M and Katalinic A. Pregnancy course and health of children born after ICSI depending on parameters of male factor infertility. Hum Reprod 2003; 18(2):351-357.

20. Tepla O, Peknicova J , Koci K, Mika J , Mrazek M and Elzeinova F. Evaluation of reproductive potential after intracytoplasmic sperm injection of varied human semen tested by antiacrosomal antibodies. Fertil Steril 2006; 86(1):113-120.

21. Noci I , Biagiotti R, Maggi M, Ricci F , Cinotti A. and Scarselli $\mathbf{G}$. Low day 3 luteinizing hormone values are predictive of reduced response to ovarian stimulation. Hum Reprod 1998; 13(3):531-534.

22. Kolibianakis E , Bourgain C , Albano C , Osmanagaoglu K, Smitz J , Van Steirteghem A, et al., Effect of ovarian stimulation with recombinant follicle- 
stimulating hormone, gonadotropin releasing hormoneantagonists, and human chorionic gonadotropin on endometrial maturation on the day of oocyte pick-up. Fertil Steril 2002; 78(5):1025-1029.

23. Ebrahim A, Rienhardt G, Morris S, Kruger T F, Lombard C J and Merwe J P. Follicle stimulating hormone levels on cycle day 3 predict ovulation stimulation response. J Assist Reprod. Genet. 1993; 10(2):130-136.

24. Zhu Y, Huang H, and Zhou F. Analysis of factors influencing the clinical in a frozen thawed embryo transfer program. Zhoughua Fu, Chan Ke Za Zhi, 2001; 36(5):290-292.

25. Ku S Y, Choi Y S, Jee B C, Suh C S , Choi Y M , Kim J G, et al. A preliminary study on reduced dose (33 or 25 micro) gonadotropin- releasing hormone agonist long protocol for multi-follicular ovarian stimulation in patients with high basal serum follicle-stimulating hormone levels undergoing in vitro fertilization-embryo transfer. Gynecol Endocrinol 2005; 21:227-231.

26. Check J H , Katsoff B , Brasile D , Choe J K and Amu $\mathbf{J}$. Pregnancy outcome following in vitro fertilization-embryo transfer (IVF-ET) in women of more advanced reproductive age with elevated serum follicle stimulating hormone (FSH) levels. Clin Exp Obstet Gynecol 2008; 35:13-15.

27. Kassab A, Sabatini L, Tozer A, Zosmer A, Mostafa M, Al-Shawaf T. The correlation between basal serum follicle-stimulating hormone levels before embryo cryopreservation and the clinical outcome of frozen embryo transfers. Fertil Steril 2009; 92:1269-1275.

28. Jong D , Eijkemans M J , Beckers N G, Pruijsten R V, Fauser B C. and Macklon N S. The added value of embryo cryopreservation to cumulative ongoing pregnancy rates per IVF treatment: is cryopreservation worth the effort? J Assist Reprod Genet 2002; 19:561-8.

29. Oehninger S, Mayer $\mathbf{J}$ and Muasher $\mathbf{S}$. Impact of different clinical variables on pregnancy outcome following embryo cryopreservation. Mol Cell Endocrinol 2000; 27(169):737.

30. Fitzmaurice J, Boylan C and Mc Clure N. Are 152 Pregnancy Rates Compromised Following Embryo Freezing to Prevent OHSS? Ulster Med J 2008; 77(3): $164-167$.

31. Pattinson H A , Hignett M , Dunphy B C and Fleetham $\mathbf{J} \mathbf{A}$. Outcome of thaw embryo transfer after cryopreservation of all embryos in patients at risk of ovarian hyperstimulation syndrome. Fertil Steril 1994; 62(6): 1192-6.

32. Coskun S, Johannes H, Saad Al-Hassan, Hamad AlSufyan, Hend Al-Mayman and Kamal Jaroudi . Day
5 versus day 3 embryo transfer: a controlled randomized trial. Hum Reprod 2000; 15(9): 9 1947-1952.

33. Mesut N, Hadar N C, Mesut A, Aksoy A, Mustafa B Cryopreservation of blastocysts is the most feasible strategy in good responder patients, Fertil Steril 2011; 5: $1121-1125$.

34. Lejeune Francoise P, Sabine V, Herbert Z, Pierre V , Nicolas H. Prospective evaluation of the optimal time for selecting a single embryo for transfer: day 3 versus day 5, Hum Reprod 2007; 88(1): 244-246.

35. Papanikolaou E G , Camus M , Kolibianakis E M , Van Landuyt L, Van Steirteghem A, and Devroey $\mathbf{P}$. In vitro fertilization with single blastocyst-stage versus single cleavage-stage embryos. N Engl J Med 2006; 354: 1139-1241.

36. El-Toukhy T, Coomara samy A, Khairy M, Sunkara $\mathbf{K}$, Seed P , Khalaf $\mathbf{Y}$ et al; The relationship between endometrial thickness and outcome of medicated frozen embryo replacement cycles. Fertil Steril 2008; 89: 832-9.

37. Singh M P, Vrotson $\mathbf{K}$ and Balen $\mathbf{A} \mathbf{H}$. Frozen embryo replacement cycle: an analysis of factors influencing the outcome. J Obstet Gynecol India 2007; 57(3):240-244.

38. Liu $\mathbf{J}$ and Huang G. Comparison of the vetrification and slow freezing protocol for day 3 embryo cryopreservation, Fertil Steril 2011;3 (5):213.

39. Noyes N, Hampton B, Berkeley A, Licciardi F, Grifo J , and Krey L. Factors useful in predicting the success of oocyte donation: a 3-year retrospective analysis. Fertil Steril 2001; 76 (9):2-9.

40. Veleva Z, Mauri O, Sinikka N, Juha S, Tapanainen L and Hannu M . Factors affecting the outcome of frozen-thawed embryo transfer. Hum Reprod 2013; 28(9): 225-243.

41. Salumets A, Suikkari A M S , Karro H , Roos A and Tuuri T Frozen embryo transfers: implications of clinical and embryological factors on the pregnancy outcome, Hum Reprod 2006; 21 (23):68 -74. 